\title{
El éxodo de los psicólogos alemanes y sus consecuencias
}

Helmut E. Lück

Universidad de Distancia-Hagen

Hagen, Alemania Federal

El autor analiza las consecuencias de la emigración de psicólogos en los años del nacionalsocialismo para la psicología alemana, así como para los emigrantes. El destino de David Katz, Kurt Lewin y Wolfgang Köhler en la emigración es asimismo comentado, señalando cómo el exilio influyó en su productividad científica. El autor estudia la reacción de William Stern ante su despido como profesor de la Universidad de Hamburgo. Por último se refiere a los años de la posguerra y el modo en que la psicología alemana se reconstituyó.

emigración / psicólogos alemanes

\section{The exodus of German psychologist and its consequences}

The author analyzes the consequences of the emigration of German psychologists in the years of the rise of Nationalsocialism and its influence both for German psychology and emigrants. Particularly, the destiny of David Katz, Kurt Lewin and Wolfgang Köhler in the emigration is reviewed, pointing out how the process of exile influenced their scientific work. The author analyzes also the reaction of William Stern after his dismissal as Profesor of the University of Hamburg. Finally, the author refers to postwar years and how German psychology was reconstituted. 
La relación de la psicología y de los psicólogos con su propia historia es problemática: con gran frecuencia $\mathrm{y}$ con orgullo suele mencionarse el nombre de grandes figuras de la psicología, pero al mismo tiempo la historia de esta ciencia como asignatura recibe un interés más bien marginal en la formación profesional respectiva. Sólo allí, donde es posible encontrar un docente que conoce sobre el tema, se dictan cursos sobre historia de la psicología, que, por lo mismo, muchas veces no es materia de evaluación obligatoria, como sí lo es por ejemplo en Estados Unidos y en España. Esto coloca a los psicólogos alemanes en la paradójica situación de reconocer que es frecuente que colegas suyos del extranjero conozcan mejor que ellos la historia de la psicología alemana.

\section{LA ABSTINENCIA EN MATERIA DE HISTORIA DE LA PSICOLOGÍA}

Un motivo para esta llamativa abstinencia -0 , tal vez deberíamos llamarla con más propiedad ignorancia- con respecto a su historia por parte de la psicología académica proviene de la propia concepción de la ciencia que predomina entre los psicólogos. Un modo de trabajo basado en los métodos de las ciencias naturales, que concede particular valor al experimento, suele arrojar al dominio de lo superado, de lo anticuado y de lo precientífico a trabajos de investigación realizados en el pasado. Eso explica por qué desde hace años la asignatura de la historia de la psicología es considerada como no necesaria en la formación de los psicólogos.

Un componente de ese programa de abstinencia fue también el hecho de que, tras la Segunda Guerra Mundial, las relaciones entre la psicología y el nacionalsocialismo apenas fueron estudiadas. Un psicólogo en formación allá por las décadas del cincuenta y del sesenta tenía apenas vaga información acerca de la psicología del ejército (Wehrmachtpsychologie), de la prohibición del psicoanálisis en los años del régimen de Hitler, y de la obligada emigración de importantes psicólogos judíos. Señalemos, de otro lado, que en algunas universidades no escapó al conocimiento de los estudiantes que una parte de la literatura de los años de 1933 a 1945 había desaparecido de las bibliotecas, porque entre los volúmenes que la conformaban tal vez se hallaban un par de incómodos escritos de alguno de sus profesores.

"Por supuesto, era para nosotros 1o más interesante en la lectura lo que había sido tachado con tinta negra, retirado con cortes de tijera, que lo que quedaba al alcance de nuestros ojos": esos son los recuerdos de quien entonces era un estudiante de psicología (Graumann, 1985).

Pero, a pesar de la curiosidad juvenil, la relación entre la psicología y el nacionalsocialismo fue apenas tratada de modo serio. Si en algunas universida- 
des éste fue un tema de discusión, esto ocurrió en parte para autojustificarse, o se circunscribió a ciertos colegas y determinadas escuelas (Leipziger Schule der Ganzheitspsychologie), con el fin de colocarlos en las cercanías de la ideología nazi.

En general, tanto los docentes como los alumnos estuvieron contentos por haber superado ese período, considerado por muchos como oscuro. Se difundió la opinión de que la investigación psicológica había padecido durante los años del nazismo: los nazis estaban en contra de la psicología, pues, por ejemplo, el psicoanálisis había sido prohibido y, además, la Wehrmachtpsychologie había sido disuelta durante el régimen de Hitler. En resumen: la psicología había experimentado un período de decadencia en esa época, del que estaba recuperándose. Albert Wellek, un influyente psicólogo, fue aún más lejos en 1960 al afirmar que la psicología incluso debía ser prohibida durante el Tercer Reich por ser una "ciencia judía" (Wellek, 1960).

Estas afirmaciones no fueron rebatidas por décadas, sino aceptadas y difundidas. Recién al comienzo de los años ochenta -esto es, 40 años después de la capitulación y mucho tiempo después del movimiento estudiantil- comenzaron a desarrollarse las primeras investigaciones de aliento que permitieron una mirada crítica a la psicología en los años del nazismo (Geuter, 1984; Graumann, 1985). Las numerosas in- vestigaciones demostraron que en torno al rol de la psicología en el nacionalsocialismo se habían difundido muchas medias verdades. La realidad histórica es -como siempre-muy compleja y hasta ahora poco estudiada.

Tras 50 años del fin de la guerra y, por tanto, con gran distancia temporal, el éxodo de psicólogos alemanes y sus consecuencias para ellos y para la disciplina deben ser vistos de modo diferenciado. En el marco del presente trabajo queremos tratar sobre la situación de la psicología académica en la República de Weimar, los efectos de la época nacionalsocialista en la psicología y los psicólogos, para lo cual nos referiremos a algunas personalidades.

\section{LA PSICOLOGÍA ANTES DE 1933}

Se suele señalar el inicio de la psicología académica en Alemania en 1879, en que el fisiólogo Wilhelm Wundt, profesor de filosofía de la Universidad de Leipzig, fundó un pequeño laboratorio de psicología en la Facultad de Filosofía. Naturalmente, tales fechas son forzadas, pues el tratamiento psicológico existía desde antes, así como también la investigación experimental.

Dos factores fueron los que favorecieron el surgimiento de la psicología experimental precisamente en Alemania: de un lado, el alto nivel de desarrollo de la fisiología, con investigadores de la talla de Hermann von Helmholtz. De otro lado, el camino triunfal de las ciencias naturales y de la medicina y, 
en especial, de la teoría de la evolución de Darwin, propagada en Alemania sobre todo por Ernst Haeckel, constituyeron poderosos estímulos para el estudio experimental de los procesos de la conciencia. Wundt se sintió atraído por este tipo de enfoque y por el tema, pudiendo apoyarse en la psicofísica de Fechner. Debe señalarse, sin embargo, que Wundt era del parecer de que los procesos más elevados de la conciencia no podían ser estudiados experimentalmente, sino que ellos eran un ámbito de estudio para lo que él llamó la Voelkerpsychologie (psicología de los pueblos), a la que dedicó varias décadas de su existencia.

Un gran número de discípulos de Wundt intentó aplicar el método experimental a problemas prácticos: Ernst Meumann en el terreno de la pedagogía; Emil Kraepelin en el de la psiquiatría; Münsterberg en el campo económico. En la correspondencia de ellos hay testimonios que evidencian que Wundt no se mostraba muy entusiasmado por tales intentos.

Nosotros solemos considerar a Wilhelm Wundt, Carl Stumpf, Oswald Külpe y William Stern como psicólogos, sin hacernos mayores problemas acerca de sus méritos en el desarrollo de la psicología aplicada. Esto no es, por cierto, un error. Pero, por otro lado, es un hecho que estos psicólogos en casi todos los casos no sólo se desempeñaban en cátedras de filosofía, sino que además se sentían filósofos. Esto vale incluso para algunos psicólogos de la generación siguiente, a la que perteneció Wolfgang Köhler.

En 1912, más de dos tercios de los filósofos que enseñaban en universidades alemanas firmaron una declaración en contra de la asignación de nuevas cátedras de filosofía a psicólogos experimentales. En realidad, fue poco lo que cambió: la psicología era entonces parte de la filosofía y como especialidad se estableció mucho tiempo después. Algo muy diferente ocurrió en los Estados Unidos donde, ya antes de la Primera Guerra Mundial, en algunas universidades (especialmente, entre las de más reciente fundación) se crearon cátedras exclusivamente dedicadas a la psicología.

Imaginémonos una típica cátedra de psicología (Psychologisches Institut) en la Alemania de la República de Weimar: un profesor, uno o quizás dos asistentes, dos o tres ayudantes y una pequeña biblioteca. Los doctorandos, por lo general no más de dos, se dedicaban a la investigación experimental de temas de la conciencia, de la percepción o del pensamiento. Así podemos perfilar la Psychologische Lehrsammlung, que fue dirigida por Traugott Oesterreich hasta 1933, año de la separación de su cargo (Oesterreich, 1954).

La atmósfera espiritual del mundo académico estaba caracterizada ya antes de la República de Weimar por la angustia ante la nivelación de la sociedad de masas, tal como ella se concre- 
tizaba en los objetivos del socialismo internacional. El típico académico de los años de Weimar tenía una actitud muy escéptica hacia el gobierno y hacia la democracia parlamentaria. Estaba dispuesto a servir al Estado, pero no a un régimen de la socialdemocracia. La percepción que él tenía de sí mismo era la de ser un nacionalista, pero en su rol de académico rechazaba cualquier participación en la política, definiéndose como "apolítico". Aquellos científi$\cos$ que no se ajustaban a este perfil eran considerados como marginales. Uno de esos marginales era Albert Einstein. No porque él fuera judío, sino por su pacifismo declarado, su internacionalismo y su simpatía por el sionismo. Su posición marginal permite explicar la facilidad con la cual el régimen nacionalsocialista pudo deshacerse de él, a pesar de que era un Premio Nobel y que gozaba de un inmenso prestigio internacional (Beyerchen, 1982).

Otro rasgo común en los intelectuales de la República de Weimar debe ser señalado: el rechazo del materialismo, un concepto que fue empleado de modo muy grueso por ellos, que lo entendían como una actitud de poco respeto ante los valores espirituales, pero también como la industrialización y los aspectos negativos de la civilización (Beyerchen, 1982). En amplios círculos de intelectuales este rechazo del materialismo iba unido a una suerte de nostalgia romántica de los valores tradicionales.
La atmósfera espiritual de la época se refleja bien en los libros de aquel entonces, que encontraron amplia acogida. Entre los más conocidos autores que trataban temas psicológicos estaban Ludwig Klages, el médico Carl Ludwig Schleich, el psiquiatra Hoche, el filólogo Richard Müller-Freienfels y el adleriano Fritz Künkel. En varios de estos autores se encuentra, aparte de una actitud crítica a la cultura de la época, un lenguaje nebuloso (en especial, en Schleich). En esa época cargada de pesimismo las ciencias naturales no tenían mucha audiencia, y tampoco la psicología experimental, contra la cual se manifestaron conocidos autores, como Eduard Spranger. La psicología espiritualista de Spranger, precisamente, fue vista como una de las prometedoras orientaciones del pensamiento psicológico y habría de permanecer como determinante en la formación de maestros en Alemania bastante tiempo después de acabada la Segunda Guerra Mundial.

Uno de los conceptos centrales de la psicología académica de fines de los años veinte fue el de la totalidad (Ganzheit), bajo el cual se agrupaban no sólo los trabajos de los psicólogos berlineses de la Gestaltheorie y los de Leipzig de la Ganzheitspsychologie, sino también los de la psicología individual de Alfred Adler. 


\section{LA PSICOLOGÍA EN LOS AÑOS DEL NACIONALSOCIALISMO}

Pocas semanas después de que Hitler asumiera el poder la así llamada Ley de Restauración del Servicio Público (Gesetz zur Wiederherstellung des Berufsbeamtentums), del 7 de abril de 1933, dispuso que los empleados públicos "de ascendencia no aria" (nicht-arischer Abstammung) debían ser pasados al retiro.

En las universidades, hasta fines del primer año del régimen nazi, 313 profesores ordinarios y 109 profesores asociados, alrededor de 400 profesores honorarios docentes privados así como 500 asistentes, instructores y ayudantes en cátedras, museos y bibliotecas fueron despedidos, un tercio de los cuales lo fue por razones raciales (Jaeger, 1993 ).

En psicología cinco del total de 15 profesores ordinarios fueron despedidos: Adhemar Gelb (Halle), David Katz (Rostock), Wilhelm Peters (Jena), William Stern (Hamburgo) y Max Wertheimer (Frankfurt). Además, Otto Selz, profesor en la Handelshochschule de Mannheim, fue también separado de su cargo. Selz emigró a Holanda y creyó estar allí en seguridad. Sin embargo, tras la ocupación de ese país, fue transportado a Auschwitz, en donde fue asesinado.

Fueron afectados por la ley los profesores asociados, como Kurt Lewin (Berlín), quien como ex voluntario del ejército alemán creyó que no sería víctima de ella, pues a pedido expreso del presidente Hindenburg la norma conte- nía un parágrafo para quienes se habían desempeñado en el frente de batalla (Frontkaempferparagraphen): quien había peleado en la Primera Guerra Mundial no debía ser despedido. Este acápite sería después retirado. Lewin se adelantó a su separación del cargo al emigrar a los Estados Unidos, pero curiosamente su nombre permaneció durante muchos años aún en el catálogo de la universidad (Vorlesungsverzeichnis), indicando que se encontraba con licencia.

En 1935 emigró Wolfgang Köhler, una de las grandes figuras de la psicología de la Gestalt, colega de Lewin y director del instituto en el cual se había desempeñado, quien además no era judío, después de que no pudiera impedir más las intromisiones y ataques de los estudiantes y autoridades nacionalsocialistas en su instituto. Köhler sería el único que daría tal paso. Él fue, asimismo, el único que formuló una apelación pública el 28 de abril de 1933 en la forma de un artículo ("Gespraeche in Deutschland") en la Deutsche Allgemeine Zeitung, en contra del despido de científicos judíos. Su protesta no provocó sin embargo ninguna reacción efectiva de solidaridad digna de mencionarse, a pesar de que su artículo recibiera más de 120 cartas aprobatorias (Jaeger, 1993).

Un destino trágico fue el del profesor asociado Kurt Huber (1893-1943), que se desempeñaba como docente en las asignaturas de métodos psicológicos. 
Su nombre forma parte de la resistencia en contra de Hitler, debido a su participación en la entrega de volantes de la Weisse Rose muniquesa. Fue condenado a muerte y ejecutado, a pesar de que en su defensa ante el tribunal destacó su ideología conservadora y nacionalista, pues, en efecto, no era un antifascista (Weber, 1993).

Después de que el gobierno de Hitler procediera a denunciar el Tratado de Versalles, se produjo una impresionante implementación del ejército y de la psicología militar. Geuter (1984) menciona las siguientes cantidades de plazas de trabajos para psicólogos en el ejército y en la marina: en 1935, 69; en 1937, 127; y, en 1938, 170.

A estas cifras hay que agregar la de los psicólogos que trabajaban en la aviación militar, que para 1942 contaba con alrededor de 150 de estos profesionales.

Si bien durante varios años del régimen hitleriano no había una clara imagen profesional de la psicología y tampoco un programa de formación específica de psicólogos, la necesidad de contar con estos profesionales por parte del ejército incentivó el establecimiento de un programa de estudios. En la forma de la norma de evaluación del diploma de psicólogo (Diplom-Prüfungsordnung für Psychologen) se dio un paso decisivo en la profesionalización de la psicología. Temas de la psicología militar se convirtieron en elementos constituyentes de la formación universitaria de los profesionales de la psicología. A su vez, esto tuvo repercusiones en la labor docente: para ser nombrado profesor de psicología en alguna universidad, la experiencia en la psicología militar (especialmente en el campo del diagnóstico) se convirtió en un punto de mucha importancia.

Entre la directiva, particularmente servicial al régimen, de la Sociedad Alemana de Psicología un número de psicólogos intentó colocar sus conocimientos y capacidades al servicio del nuevo sistema. Como ejemplo puede mencionarse a Gerhard Pfahler (18791976), cuya carrera universitaria experimentó un ascenso meteórico: Pfahler fue profesor de psicología en Giessen y rector de la universidad (tal vez el más joven rector en toda Alemania). Pero a sus funciones como rector, Pfahler -amigo de Rudolf Hess-, agregó las de Sturmführer (jefe de la fuerza de choque) de la SS. En esos años Pfahler decidió vincular su tipología ecléctica de la personalidad (que había desarrollado mucho antes) a la doctrina racista del nacionalsocialismo. En 1938 Pfahler pasó a desempeñarse como profesor de psicología y pedagogía en la renombrada Universidad de Tubinga.

Aunque fueron muchos los psicólogos que se ofrecieron a colaborar con el régimen, hasta ahora no se ha podido establecer la participación de ellos en el trabajo de propaganda, en el proceso de deportación o en los campos de concentración. Esto último tiene tal vez 
que ver con el nivel de desarrollo de la psicología como ciencia y como profesión: no existía en ese entonces la psicología de la publicidad y de la propaganda, el instrumental de la investigación de campo (como las encuestas) se encontraba en un estado de incipiencia, y la misma psicología clínica contaba con un reducido y no muy consistente repertorio de posibilidades diagnósticas y terapéuticas. Es probable que debido a esto la psicología apareciera a los ojos de los gobernantes como una disciplina de poca importancia y utilidad para sus proyectos .

En todo caso, en contraste con la pérdida en recursos humanos y la disminución en la calidad de la investigación, en la psicología se produjo, en varios aspectos, una elevación cuantitativa. Durante los años del nacionalsocialismo se establecieron los institutos de psicología, se crearon nuevas plazas de profesores de la especialidad y otras, que no correspondían a esta especialidad, fueron transformadas en puestos de profesores de psicología.

Sin embargo, en la vida académica se establecieron algunas circunstancias peculiares. Algunas revistas psicológicas (sin que hubiera regulación legal alguna de por medio) exigieron que en las referencias bibliográficas de los artículos se colocara un asterisco al lado del nombre de autores judíos. Esto llevó a que muchos renunciaran a hacer citas de psicólogos judíos con el fin de poder ver impresos sus trabajos. Sin embargo, era posible citar a estos psicólogos, como lo demuestra el hecho de que uno de los más citados en la época del nacionalsocialismo fuera David Katz, quien desde 1933 se encontraba en el exilio. Al mismo tiempo, muchos psicólogos evitaron citar a aquellos colegas, como Jaensch, que de modo muy evidente se habían acercado al nacionalsocialismo (Lange, 1990).

En 1942 los departamentos de psicología de la aviación y del ejército fueron disueltos. Esto llevó a que después de la Segunda Guerra Mundial se argumentara que la psicología fue una disciplina perseguida por los nacionalsocialistas. La afirmación puede ser considerada, no obstante, como falsa. La razón principal para la disolución debe verse en el hecho de que un pronóstico diferencial de las habilidades de los soldados ya no era más necesario. Las pérdidas en el ejército y en la aviación eran tan grandes, que la selección de éstos resultaba una tarea carente de sentido.

\section{ACerCa de la situación especial DEL PSICOANÁLISIS}

En lo que se refiere a la prohibición del psicoanálisis, es necesario ofrecer una perspectiva más diferenciada de lo sucedido. Es cierto que las obras de Sigmund Freud fueron lanzadas al fuego el 10 de mayo de 1933 con el así llamado lema "Contra la sobrevaloración de la vida sexual destructora del espíritu, y por la nobleza del alma hu- 
mana", y que Freud, así como muchos de sus discípulos judíos, después de la anexión de Austria, tuvieron que partir al exilio a fin de ponerse a buen recaudo de los nazis. Es cierto, también, que el Instituto Psicoanalítico de Viena y sus instalaciones fueron liquidados y que el psicoanálisis fue oficialmente prohibido.

Pero no es cierto, sin embargo, el que el psicoanálisis como terapia no pudiera ser practicado en el Tercer Reich. Después de la disolución del Instituto de Psicoanálisis se creó en Berlín (en 1936) el Deustche Institut für Psychologische Forschung und Psychotherapie (Instituto Alemán de Investigación Psicológica y Psicoterapia), cuya tarea era el desarrollo de una Seelenheilkunde alemana (una cura de almas alemana). En ese instituto se abarcaron las diferentes corrientes de la psicología dinámica, las que fueron practicadas bajo la dirección médica. De ahí que la proclamada por los psicoanalistas destrucción del psicoanálisis deba ser considerada más bien una leyenda (Lockot, 1985). Sí es verdad, en efecto, que el psicoanálisis perdió en esos años a muchos de sus principales representantes y comenzó un proceso de provincialización, que sólo pudo ser superado mucho después de la Segunda Guerra Mundial gracias al trabajo de Alexander Mitscherlich, con el cual volvió a ganar nivel en los estándares internacionales de la investigación.

Un tema particularmente espinoso referido al psicoanálisis en los años del hitlerismo es el del rol de Carl Gustav Jung, quien -probablemente estimulado por los largos años de disputa con Freud- creyó reconocer en esa época una oportunidad para la difusión de su psicología analítica. Una serie de declaraciones suyas (como la de que el potencial del inconsciente ario era mayor que el del inconsciente judío) puede haber parecido plausible en aquella época, pero son en realidad difíciles de disculpar. La cercanía de Jung a Alemania en esos años le valió el rechazo de muchos en Suiza, país en el cual vivía (Lockot, 1985) .

\section{TRES EMIGRANTES EN COMPARACIÓN CUANTITATIVA}

Si se quiere describir el destino de los emigrantes y establecer las semejanzas entre ellos hay un procedimiento que, aunque parezca trivial, ofrece alguna información de significado: el análisis cuantitativo de sus trabajos. Comparemos a tres emigrantes (que se encontraban más o menos en la misma edad), David Katz, Kurt Lewin y Wolfgang Köhler, con referencia a la cantidad y naturaleza de sus publicaciones. Eso nos permitirá encontrar algunas diferencias de interés (Lück \& Clever, 1991).

En el caso de David Katz, éste, en la emigración en Suecia, aborda nuevamente una serie de cuestiones que ya había trabajado en Alemania. Al mismo tiempo se aboca a nuevos temas y problemas, y publica una serie de trabajos 
sobre tópicos de psicología general y de los procesos cognitivos superiores. En su caso, la productividad continúa semejante y con una gran variedad de temas.

Si se considera la elevación del número de publicaciones, que después de la emigración son exclusivamente en inglés, y el tratamiento de un nuevo tema: la psicología social, entonces Kurt Lewin aparece como el que mejor se adapta a la cultura norteamericana, de modo tal que bien se puede hablar de una segunda carrera en su caso (Schoenpflug, 1991; Lück, 1996).

Wolfgang Köhler, en contraste con Lewin y Katz, publicó mucho menos en la emigración, y su temática no experimentó casi ningún cambio.

En los tres autores podemos encontrar una clara interrupción en la productividad en la fase inicial de la emigración, pero también una exitosa continuación de sus carreras científicas, aunque en formas diferentes. Incluso esta comparación tan gruesa permite afirmar que no existe un destino típico de los psicólogos en el exilio. La productividad continuada que hemos registrado en Katz, Lewin y Köhler no es una característica de todos los psicólogos. La más triste demostración de esto la constituye el destino de Karl Bühler, cuya contribución a la psicología del lenguaje fue muy importante. Su esposa, Charlotte Bühler, tenía cierta ascendencia judía y ambos dirigían con energía y éxito el Instituto de Psico- logía de Viena. Tras la anexión de Austria por parte de los nazis no quedó otra posibilidad a los Bühler que emprender el camino de la emigración, primero a Suecia y de ahí a los Estados Unidos, donde ambos tuvieron grandes dificultades para establecerse. Las pocas plazas docentes en los difíciles años de la crisis económica estaban ya ocupadas por emigrantes alemanes que habían llegado antes que ellos. Mientras Charlotte Bühler pudo avanzar profesionalmente y llegó a ser una de las fundadoras de la psicología humanística, Karl Bühler postuló sin éxito a nuevas posiciones laborales. En esos años preparó algunos pequeños trabajos sobre temas biológicos, que no fueron publicados; ningún trabajo suyo de importancia dedicado a la psicología aparecería ya.

\section{El ejemplo de William Stern}

William Stern es conocido entre los estudiantes de psicología como el creador del cociente intelectual. Pero su aporte a la psicología se extiende en realidad mucho más allá: la psicología del desarrollo, la psicología diferencial, el psicodiagnóstico, la psicotécnica (el concepto proviene de él), la psicología del testimonio; todos esos fueron campos de trabajo de investigación suya. Él fue uno de los iniciadores de la Universidad de Hamburgo y condujo al Instituto de Psicología de esa casa de estudios a un elevado nivel de prestigio internacional. 
Stern fue en 1904 uno de los miembros fundadores de la Sociedad de Psicología Experimental; desde 1921 perteneció a la directiva, en 1929 fue su director ejecutivo, y en 1931, en la asamblea general en el congreso que se celebraba en Hamburgo fue elegido su presidente. Para entonces la sociedad se denominaba Sociedad Alemana de Psicología (como se sigue llamando aún hoy). Con esa nominación Stern había alcanzado la cima de su carrera académica. Con motivo de sus 60 años, 14 días después del congreso de Hamburgo, Stern recibiría un volumen de escritos en su homenaje.

En el otoño de 1933, es decir sólo dos años después, la Sociedad Alemana de Psicología se reunió en Leipzig. El nombre de Stern había sido retirado de la lista de miembros de la institución. El nuevo presidente de la Sociedad, Felix Krueger, ni siquiera lo mencionó por su nombre al momento de pronunciar su discurso. Stern no podía ingresar a su Instituto en Hamburgo y las revistas y colecciones editoriales que él editaba fueron suspendidas o, a pesar de su enfática protesta, asumidas por otras personas.

En muchos científicos judíos la emigración y el nacionalsocialismo condujeron a una clara expresión de su propia identidad judía. Ése fue el caso del físico Max Born, quien en una carta a Albert Einstein en abril de 1933 le expresó que él recién experimentaba su identidad de judío, algo que hasta entonces no había sido mayormente vivenciado (Beyerchen, 1982).

¿Cómo se vinculaba Stern con su propia condición de judío? Si se observa la gran cantidad de sus publicaciones no es posible reconocer (en oposición a lo que sucede con Katz y Lewin) en título alguno, una consideración especial por parte suya de temas especiales de la cultura judía, la educación o la mitología judías. Esto es: Stern no puede ser incluido entre la lista de autores judíos, si por ellos se entiende a quienes de manera especial trataron temas vinculados con la problemática judía. Ya en 1915 Eduard Spranger, en relación con el proceso de seleccionar a Stern como profesor en la Universidad de Hamburgo, había escrito: "Si él no es todavía profesor esto se debe evidentemente a que es judío. Pero su condición de tal en modo alguno se manifiesta como algo negativo, tal como lo afirman quienes lo conocen. Si se quiere ser plenamente objetivo, esto no debería ser tomado en cuenta" (citado según Moser, 1988).

La asimilación a la cultura alemana por parte de Stern no fue, sin embargo, plena. Por propia convicción Stern no llegó tan lejos como para proceder a recibir el bautizo cristiano. A la pregunta acerca de por qué esto no sucedió, su hijo, Günther Anders, escribe:

Ésa es una pregunta justificada. Esto es no sólo para usted, no-judío, como para mí difícil de comprender. Pues él, a pesar de su distanciamiento del judaísmo, 
nunca tuvo como idea la de renunciar a su condición de judío (fuera lo que fuera lo que él entendiera por ella). Le habría parecido un precio indigno para posibles cargos honoríficos. En efecto, él tuvo la oferta de ser Profesor de Psicología en la Universidad de Berlín, a cambio de que él cumpliera con una 'pequeña formalidad' [el bautizo; N. del T.]; algo que él rechazó de modo terminante. Usted puede darse cuenta: las cuentas no estaban saldadas. Un mínimo de principios sobre el particular quedó en él. Desarrollar perspectivas sobre esa situación era algo que él no podía permitirse. Su imagen del mundo (que se derrumbó en 1933) no podía él ponerla en tela de juicio. La llegada del nacionalsocialismo no sólo no fue presentida por él, sino que inclusive la sometió a un proceso de represión positiva (Anders, 1979; Michaelis-Stern, 1972).

Es decir: Stern conservó un resto de su condición de judío, que él no abandonó. Pero también adoptó posiciones antisionistas, que se expresan en una carta, del 25 de marzo de 1928, a su amigo Cohn, cuando escribe sobre su hija Eva, indicando que ella tiene un "inextricable impulso hacia Zion" (Lück \& Lowisch, 1994).

Mucho más dramático lo formula la misma Eva Michaelis-Stern:

Mis padres (...) estaban mortalmente disgustados por mi sionismo y porque yo formara parte de un movimiento de juventudes sionistas y que quisiera ir a Palestina. Todo esto iba en contra de lo que ellos podían comprender y de las esperanzas que tenían acerca de mí (Michaelis-Stern, 1989).

Si William Stern en realidad reprimió positivamente - como lo señala su hijo- la llegada del nacionalsocialismo, es algo que no se puede determinar con precisión. Pero, comparando su comportamiento con otros psicólogos judíos, como Kurt Lewin -que mantenía una amistad cercana con la familia Stern- llama la atención cuán vacilantemente reaccionó Stern ante el régimen nazi y cuánto tiempo necesitó para decidirse a emigrar. Si bien Stern no era un apolítico, no cabe duda que lo sorprendió la radicalidad del régimen nazi. El hijo de Stern escribe:

Mi padre estaba completamente desconcertado frente a todo aquello que sucedió. Cuando de un día a otro fue separado de sus labores docentes, de la redacción de sus revistas, y de su bastante bien equipado Instituto de Psicología en Hamburgo, su mundo se derrumbó (Stern-Anders, 1971).

El 1 de setiembre de 1933 -un poco antes de la "Noche de los Cristales" (Reichskristallnacht)- Stern escribió (con formulaciones más o menos vagas) a su amigo Cohn sobre su situación en la Universidad de Hamburgo y el despido de profesores judíos:

Nosotros tenemos poca disposición a ver estas metamorfosis de cerca, pero hasta ahora está claro que en diciembre o enero abandonaremos nuestra casa. Todo lo demás es impreciso. De una manera vaga aparecen en el horizonte posibilidades de conferencias por invitación en oriente y en occidente, pero ellas ocuparían sólo unos cuantos meses. En la actualidad es realmente imposible hacer planes a largo plazo (Lück \& Lowisch, 1994). 
Stern y sus colaboradores judíos fueron despedidos. Su asistenta (no-judía) Martha Muchow se suicidó el 29 de setiembre de 1933 (es decir, pocos días después de la carta a la que nos hemos referido en el párrafo anterior), como resultado de la profunda impresión que le había causado la ola de despidos y represalias. Todavía otra persona, cercana a Stern, con quien había trabajado muchos años, se suicidaría: el psicólogo Otto Lipmann.

Cercano ya a pasar a la condición de emeritus, emigra Stern con su esposa primero a Holanda, donde él podrá publicar en alemán su Allgemeine Psychologie auf personalistischer Grundlage (Stern, 1935). Stern conservó su residencia en Hamburgo, en parte para no perder su derecho a una pensión que cada vez iba perdiendo su valor. Varias veces viajó a Hamburgo. Después, cuando se hizo imposible el retorno a Alemania, emigró a los Estados Unidos, en donde dictó clases en inglés y se dedicó al desarrollo de su sistema filosófico, que a muy pocos interesaba. Así, Stern terminó convertido en los Estados Unidos, como correctamente lo anota su discípulo Gordon Allport, en un "monumental defensor de una cuestión impopular". Stern le escribiría a su colega Katz, en enero de 1938, pocas semanas antes de su muerte:

No sé si a Usted le va como a nosotros: el destino del envejecido y maltratado resto de los que se han quedado en Alemania y los acontecimientos en Polonia, en Rumanía, etc., nos pesan tanto, que uno no puede disfrutar la relativa tranquilidad en la que se encuentra, a pesar de lo agradecido que se pueda estar por ella (Lück \& Katz, 1995).

Stern murió el 27 de marzo de 1938, aún relativamente joven, en Durham, North Carolina. Clara Stern, su esposa, vivió aproximadamente una década más.

\section{LA SITUACIÓN DESPUÉS DE LA CAPITULACIÓN}

Muy prontamente después de la capitulación, las universidades reiniciaron su vida académica por cierto bajo condiciones bastante precarias. Muy pocos profesores de psicología fueron separados en el proceso de desnazificación. Entre ellos estuvo el ya mencionado Pfahler, quien fue despedido en octubre de 1945, para ser enviado al retiro en 1948 aunque conservando sus derechos de enseñanza. En 1952 retorna a la actividad docente; en 1953 queda habilitado para la enseñanza superior. En 1959 pasa a la condición de emérito y continúa con su labor docente hasta 1964.

A mediados de los años cincuenta la mayoría de las cátedras de psicología en Alemania estaban ocupadas por psicólogos de la Gestalt o de la Ganzheitspsychologie. Esto significa una continuidad en lo personal y en el contenido de la enseñanza del estado de cosas de la época del nazismo. La oportunidad para un nuevo inicio quedó, así, fuera 
de lugar. Una modificación digna de ser mencionada (bajo el influjo del conductismo) en la psicología alemana ocurrió recién con la siguiente generación de profesores a fines de los cincuenta y comienzos de los sesenta.

Característico de esta época de la posguerra es, asimismo, que sólo muy pocos de los psicólogos expulsados por el nazismo volvieran a sus puestos: Traugott Konstantin Oesterreich volvió a su puesto de profesor en Tubinga; Hamburgo llamó al discípulo de Stern, Curt Bondy. En algunos casos se restablecieron antiguos contactos: Bondy llamó a Katz de Goeteborg a Hamburgo a fin de que diera un ciclo de conferencias como profesor honorario; Wolfgang Köhler visitó la Universidad Humboldt -como se llama la Universidad de Berlín desde 1946- así como la Freie Universitaet, evidentemente con sentimientos muy ambivalentes, pues temía - con toda razón- encontrarse con personas que habían tomado parte en el proceso de destrucción del instituto que él había dirigido.

Por último, otro rasgo de la psicología de la posguerra es la represión de lo sucedido. Eso ha llevado a que sólo en los últimos años se hayan aclarado algunos temas. Así, por ejemplo, se supo hace algunos años que la Universidad de Hamburgo, basándose en el retiro de los derechos de nacionalidad de sus graduados judíos, casi en un mismo trámite, les retiró el grado de doctor. Entre ellos se encontraban varios discí- pulos de Stern, que se habían doctorado con gran prisa en el verano de 1933. La mayoría de los emigrantes probablemente a lo largo de su vida no supieron que mientras vivían en el exilio se les había despojado de su grado de doctor en Alemania. Debe decirse, por cierto, que la Universidad de Hamburgo en los años recientes ha trabajado intensamente y con honestidad en la clarificación y comprensión de su propio pasado.

\section{ConClusión}

Mirado de cerca, el éxodo de psicólogos alemanes durante los años de Hitler se nos presenta como un fenómeno más complejo de lo que se suponía. Esto se debe en parte a factores de corte biográfico y a actitudes de cada uno de sus protagonistas. La emigración forzada abrió las puertas a unos (Lewin) para una segunda carrera, pero a otros los llevó al silencio (Karl Bühler).

Desde la perspectiva de la comparación internacional la psicología en los años del nazismo perdió en calidad y perdió, asimismo, sus vínculos con los estándares internacionales. Un proceso de asimilación total al nazismo no ocurrió, sin embargo. La emigración de figuras como Lewin, Selz, Stern, Köhler y Wertheimer es probablemente la causa de esa pérdida de nivel. El progreso material de la psicología en esos años aceleró su proceso de profesionalización en dirección al psicodiagnóstico. El régimen de formación y gradua- 
ción de psicólogos que existe desde 1941 y que permanece casi inmodificado es una evidencia de la continuidad de una situación surgida en los años del nacionalsocialismo.

La hipoteca que pesa sobre la psicología como disciplina y con la cual ella debe enfrentarse es un poco diferente a la de otras disciplinas y se puede resumir en unos cuantos términos más bien generales: la ausencia de resistencia en contra de la política racial del nazismo; el servilismo de los representantes de la psicología hacia el nuevo régimen; la emigración de figuras destacadas, $y$, después de la guerra, la negativa a conocer los aportes que los emigrantes habían logrado en sus años de exilio. A estas pesadas hipotecas hay que agregar el hecho de que el desarrollo de la psicología como profesión guarda estrecha vinculación con la psicología militar del régimen de Hitler. La influencia "modernizadora" de los años del nacionalsocialismo en el sentido social (Dahrendorf) y en la psicología como profesión han contribuido a que no se produjera un nuevo inicio: hasta los años sesenta se puede observar una continuidad en el ámbito de las personas y de los contenidos determinantes de la psicología alemana.

Se dice que cada generación debe escribir de nuevo la historia. Nosotros tenemos la oportunidad de mirar con perspectiva crítica los efectos del nacionalsocialismo en la psicología, con una libertad mucho mayor que la que tuvo la generación anterior.

\section{REFERENCIAS}

Anders, G. (1979). Wenn ich verzweifelt bin, was geht's mich an. En: Günther Anders im Gespraech mit Matthias Greffrath. Zerstoerung einer Zukunft. Gespräche mit emigrierten Sozialwissenschaftlern, Reinbeck bei Hamburg: Rowohlt.

Beyerchen, A. D. (1982). Wissenschaftler unter Hitler. Physiker im Dritten Reich. Köln: Kiepenheuer \& Witsch.

Geuter, U. (1984). Die Professionalisierung der deutschen Psychologie im $\mathrm{Na}$ tionalsozialismus. Frankfurt: Fischer.

Graumann, C. F. (ed.), Psychologie im Nationalsozialismus, Berlin, SpringerVerlag.

Graumann, C. F. (1985). Psychologie im Nationalsozialismus. Eine Einführung. En: Graumann, C. F. (ed.), Psychologie im Nationalsozialismus, Berlin, Springer-Verlag, 1-13.

Jaeger, S. (1993). Zur Widerständigkeit der Hochschullehrer zu Beginn der nationalsozialistischen Herrschaft. Psychologie und Geschichte, 4, 219-228.

Lange, L. (1990). Externe Einflüsse auf die Wissenschaft und die Reaktion der 'wissenschaftlichen Gemeinschaft' am Beispiel von E. R. Jaensch und der Zeitschrift für Psychologie 1933-1944. Zeitschrift für Psychologie, 198, 121136.

Lockot, R. (1985). Erinnern und Durcharbeiten. Zur Geschichte der Psychoanalyse und Psychotherapie im Nationalsozialismus. Frankfurt: Fischer.

Lück, H. E. \& Katz, Th. (1995). Vertriebene Psychologen im Gespräch: Ein bemerkenswerter Brief von William 
Stern an David Katz. Psychologie und Geschichte, 7, 141-155.

Lück, H. E. (1996). Die Feldtheorie und Kurt Lewin. Weinheim: Beltz PVU.

Lück, H. E. \& Clever, L. (1991). Quantitative Werkanalyse am Beispiel Katz, Lewin und Köhler. En: Lück, H. E. \& Miller, R. (eds.), Theorien und Methoden psychologiegeschichtlicher Forschung, Goettingen, Hogrefe, 125-139.

Lück, H. E. \& Lowisch, D.-J., eds. (1994). Der Briefwechsel William Stern - Jonas Cohn. Dokumente einer Freundschaft. Frankfurt: Peter Lang.

Michaelis-Stern, E. (1972). William Stern 1871-1938. The man and his achievements. Year Book XVII, Leo Baeck Institute, 143-154.

Michaelis-Stern, E. (1989). 'Eva: Besonders resistent gegen Suggestionen'. Entrevista con Eva Michaelis-Stern realizada por Angela Graf-Nold. Psychologie heute, 16 de julio, 62.

Moser, H. (1988). Die verlorene Psychologie des William Stern. Hamburgo: Manuscrito inédito.

Oesterreich, M. (1954). Traugott Konstantin Oesterreich. "Ich"-Forscher und Gottsucher. Lebenswerk und Lebensschicksal. Stuttgart: Frommann.

Schoenpflug, W., ed. (1991). Kurt Lewin Person, Werk, Umfeld. Frankfurt: Peter Lang.

Stern, W. (1935). Allgemeine Psychologie auf personalistischer Grundlage. La Haya: Nijhoff.

Stern-Anders, G. (1971). Geleitwort zur 7. Auflage. En: Stern, W., Psychologie der frühen Kindheit bis zum sechsten
Lebensjahr, Heidelberg, 10. ed., Heidelberg: Quelle und Meyer.

Weber, K. (1993). Kurt Huber. En: Lück, H. E. \& Miller, R., eds., Illustrierte Geschichte der Psychologie, Munich, Quintessenz, 185-187.

Wellek, A. (1960). Deutsche Psychologie und Nationalsozialismus. Psychologie und Praxis, 4, 177-182. 